\title{
Dynamic Modeling of Metal Turning Elements of Mechanical Processing
}

\author{
Madissoo, M.; MAKSAROV, V.; AraK, M. \& OlT, J.
}

Abstract: Mechanical processing of materials is critical to assessing the quality of processing accuracy. For machine elements determining the dimensions and tolerances are the most important dimensions of their surfaces, which work together. The shape- and position tolerances may raise the existence of failures in the performance and mechanisms of agricultural machinery. We hereby propose a method for the construction of small dimensional dynamic models, on the basis of which the modelling of the technological systems of mechanical processing is accomplished. It is necessary for increasing the manufacturing precision of these components.

Key words: metal machining, deformation, cutting, chatter vibrations
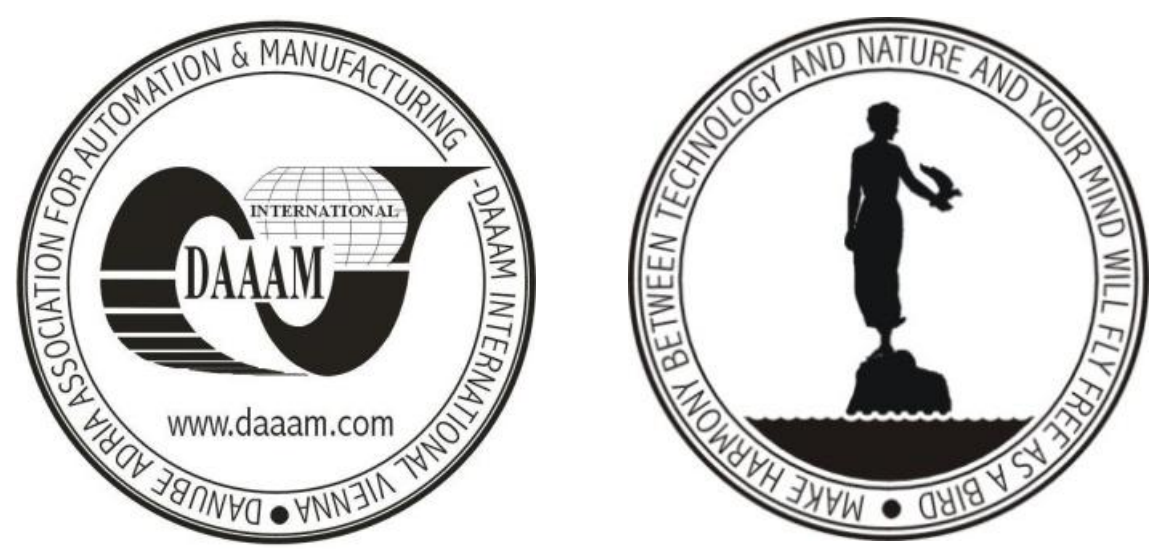

Authors' data: $\mathrm{PhD}$ student. Madissoo, M[arten]*; Prof. Maksarov, V[iacheslav]**; PhD student. Arak, M[argus]*; Prof. Olt, J[ueri]*; *Estonian University of Life Science, Institute of Technology, Kreutzwaldi 1a, 51014, Tartu, Estonia, **National Mineral Resources University «Mining University», Vasilevsky Island, the House 2, 199106, St.-Petersburg, Russia, marten.madissoo@emu.ee, dmmd@emu.ee, margus.arak@emu.ee,jyri.olt@emu.ee

This Publication has to be referred as: Madissoo, M[arten]; Maksarov, V[iacheslav]; Arak, M[argus] \& Olt, J[ueri] (2012). Dynamic Modeling of the Cutting Process, Chapter 18 in DAAAM International Annals \& Proceeding 2012, pp. 213-224, B. Katalinic (Ed.), Published by DAAAM International, ISBN 978-3901509-86-5, ISSN 1726-9679, Vienna, Austria

DOI: $10.2507 /$ daaam.scibook.2012.18 
Madissoo, M.; Maksarov, V.; Arak, M. \& Olt, J.: Dynamic Modeling of Metal Tur...

\section{Introduction}

For the definition of the characteristics reflecting the dynamic quality of a cutting system it is necessary to construct the boundaries of its region of stability in the turning process. In this task we include the following stages:

- construction of the dynamic model for a technological cutting system that is a closed potentially auto-oscillatory system allowing for the maximum possible completeness of inert and elastic-dissipative properties in the technological system;

- passage to a simplified dynamic model, equivalent to the relatively spectral characteristics of the initial one (refined multidimensional) applying the substantiated criterion of proximity in the assigned frequency range;

- construction in the space of parameters of the following limits for the region of stability: solid;

a) upper maximum - built on the assumption that the workpiece is absolutely

b) lower maximum built with the use of static characteristics in the system of basing - fastening.

The relative position of the upper and lower maximum boundaries determine the reserve for an increase in the dynamic quality to perform control of the metal processing system. This research deals with the identifying of possible solutions for the tasks mentioned above.

\section{Materials and methods}

The properties of a turning system are defined by its static and dynamic characteristics. The most important static characteristics are the stiffness of the main holding systems, their geometric accuracy and the errors in sizes and form determined by them in the quasi-static regimes of working. Dynamic characteristics determine the zones of stability of the cutting process, the spectral composition and level of amplitudes of oscillating processes, dynamic errors in sizes and form of products, and also the properties of the surface layer (Stephenson, 2006; Podporkin, 1959).

For the analysis of the dynamic properties of the processes involved in the cutting of materials it is necessary to construct a mathematical model of the system. The selection of the schematic of this model is concerned, first of all, with the isolation of subsystems and specification of the structure of connections between them, the determination of the number of degrees of freedom and type of generalized coordinates, necessary for the complete description of processes present in the system (Murashkin, 1977; Veitz, 1972).

As a rule, it is the intuitive-topological method that is used for the development of models of carrier systems. The essence of this method consists in the breakdown of the carrier system into separate inert blocks, which correspond to the models of an absolute solid. It is assumed that the blocks are interconnected with elastic (or elasticdissipative) joints. The totality of such subsystems of the carrier system forms a 
three-dimensional and according to some assumptions a flat dynamic model of the system (Murashkin, 1977; Kudinov, 1967; Eliasberg. 1993).

It is known (Kudinov, 1967; Veitz \& Marõnenko, 1972) that metal-working machines are essentially nonlinear discrete continuous dynamic systems, the precise mathematical description of which by a model is a challenge. However, knowing that the elastic and dissipative properties of a technological system are determined in essence by deformations and damping of joints, it is possible to devise a simplified dynamic model of a machine tool for practical calculations (Stephenson, 2006; Kudinov \& Blinov, 1974). Furthermore, with the composition of the dynamic model of a processing system according to the basic concept of the vibration theory (Eliasberg \& Binder, 1989; Veitz \& Martõnenko, 1972; Panovko, 1960), it is possible to limit the degrees of freedom to a finite number when the dynamic properties of a system are examined in the limited range of frequency.

The basic parameters of the discrete dynamic model of an oscillatory system include (Murashkin, 1977; Kudinov \& Blinov, 1974): reduced masses m, stiffness coefficients $c$, coefficients of force $b$ and of moment $b_{\varphi}$ of linearized resistances. For determining the parameters indicated above the axes of the three-dimensional system of coordinates of a technical system for the process of grinding were oriented as follows: $z$ axis coincides with the direction of longitudinal feed, axis $x$ coincides with the direction of cross feed, and $y$ axis is perpendicular to plane $x-z$. According to the studies (Murashkin, 1977; Kudinov \& Blinov, 1974, Panovko, 1960, Ozel, 2006), carried out in the field of the vibration theory of technological systems, it is possible to assume that the fluctuations in different directions are not interrelated.

Fig. 1. presents the phenomenological model of the system. The determinations of all parameters of the system were carried out in accordance with the established procedure. Natural frequencies and logarithmic decrements of the linearized resistances were determined by the method of free chatter (Murashkin, 1977). The determination of the stiffness coefficients of the machine tool units, which coincide with the direction of the action of the force components of cutting, were performed with the static method (Sokolovski, 1958; Kovačič, 1998).

The value of reduced mass $i$ of that element of the system $m_{i}$ is determined from the formula:

$$
m_{i}=\frac{c_{i}}{\omega_{i}^{2}}
$$

where $c_{i}$-stiffness coefficient, $\mathrm{N} \mathrm{m}^{-1} ; \omega_{i}-$ the intrinsic angular frequency, $\mathrm{s}^{-1}$.

Force coefficient of the linearized resistance $b_{i}\left(\mathrm{~N} \mathrm{~s} \mathrm{~m}^{-1}\right)$ is equal to (Murashkin, 1977):

$$
b_{i}=2 \lambda_{i} \cdot f_{c i} \cdot m
$$


where $\lambda_{i}$ - logarithmic decrement is $i$-th element of the system; $f_{c i}$ - the natural frequency of the element, Hz. For the torsional system the given moment of inertia is determined $J_{\varphi}\left(\mathrm{kg} \mathrm{m}^{2}\right)$ :

$$
J_{\varphi}=\frac{c_{\varphi}}{\omega_{\varphi}^{2}}
$$

where $c_{\varphi}$ - the coefficient of torsional stiffness of the element, $\mathrm{N} \mathrm{m} \mathrm{r^{-1 }} ; \omega_{\varphi}-$ the angular frequency of the natural torsional oscillations, $\mathrm{s}^{-1}$.

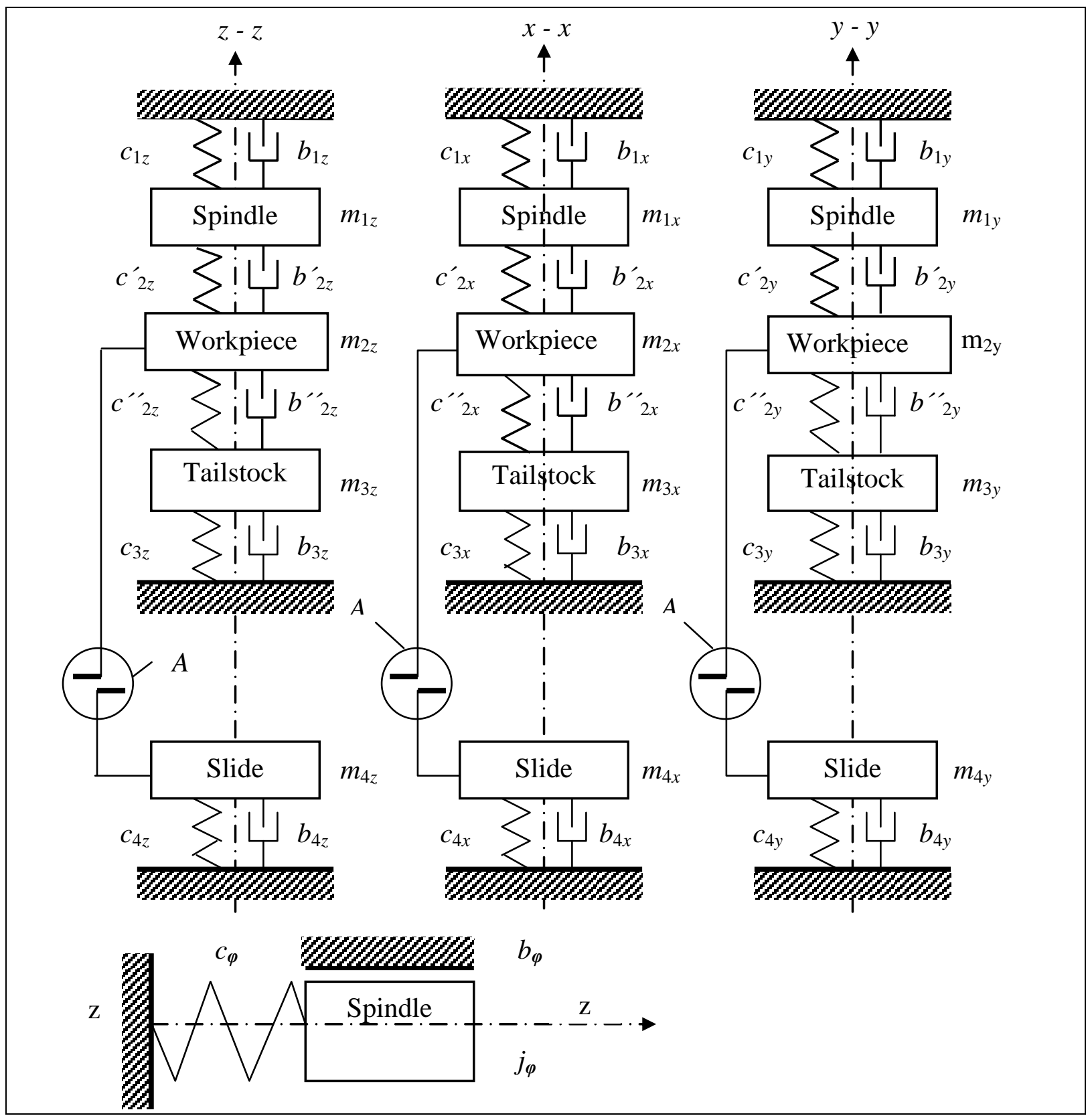

Fig. 1. The phenomenological model of the system, where A - denotes the arbitrary symbol of power interaction of the subsystem 
Coefficient of the moment of linearized resistance $b_{\varphi}(\mathrm{N} \mathrm{m} \mathrm{s})$ is determined from the formula (Murashkin, 1977):

$$
b_{\varphi}=2 \cdot \lambda_{\varphi} \cdot f_{\varphi} \cdot J
$$

where $\lambda_{\varphi}$ - the logarithmic decrement of the natural torsional oscillations; $f_{\varphi}$ - the natural frequency of torsional oscillations, $\mathrm{Hz}$.

\section{Theoretical results}

For the analysis of the dynamic properties of a processing system, and also specifying the capabilities to control the process of cutting, it is necessary to switch over from the complex system to a simplified model. In case of mechanical processing the dynamic model of a turning system can be represented as a complex system, which is organized hierarchically and is goal-directed to function as the totality of a large number of elements connected in terms of information and interaction (Murashkin, 1977; Ozel 2006).

The groups of elements are combined into blocks and they are considered as subsystems of a complex system. Such subsystems include items like the machinefixture-tool-workpiece (MTFW) (Li et al., 2010). Although the dynamic properties of separate subsystems can be fully investigated sufficiently, obtaining a satisfactory global model of a turning system is a very complicated task. That is explained by the specific manifestation of the integrative property, which is inherent in the system as a whole and which is characterized by the complex structure of connections and a variety of information involved in the interaction of the subsystems. The chief characteristic is the fact that it is autonomous and closed. Actually, the mount system interacts with the tool through the fastening system. The shaping motions are communicated to the tool when it interacts with the workpiece. As a result of this interaction forming of the work-piece is performed. The work-piece interacts with the mounting system of the machine tool through the fastening system, which is the fixture of the machine-tool. The independence of a technological processing system is conditional, since in certain cases it is necessary to consider the interaction of the external sources, through the foundation, with the mounting system of the machine tool (Olt et al., 2011).

Depending on the relationships of space, in which the working process is performed, which determines the interaction between the elements of a turning system, it is possible to describe this process in terms of the dependence of forces of interaction on the displacement of elements (Murashkin, 1977; Kudinov, 1967).

The coordinates, which describe the above mentioned displacements, are the output coordinates of the element of the particular system and the input coordinates of the corresponding working process. Under specific conditions the dynamic special feature of the process is responsible for the fact that the corresponding forces, induced by displacement, support or distribute the energy, reported by the external source. A change in the conditions for the interaction of forces occurs. This energy 
Madissoo, M.; Maksarov, V.; Arak, M. \& Olt, J.: Dynamic Modeling of Metal Tur...

transfer in the closed cycle leads to a change in the dynamic states, which can become unguided. The dynamic characteristics of the mounting system of the machine tool in this case, in fact, appear to be predefined and are determined, as a rule, by the experimental-design method accounting for its current state (Kudinov \& Blinov, 1974).

The self-oscillatory system of mechanical processing is principally nonlinear. At the same time, disregarding the nature and characteristic features of nonlinearities, let us note that many practically important problems of the dynamics of a turning system can be solved in the linear (linearized) setting. Since the self-oscillatory work mode of metalworking equipment, as a rule, are not included among the operational ones, the approximate determination of the limits of the stability region of the system on the basis of the linearized model is essentially important. Therefore, from among the existing methods of approximate description it is possible to select the operator of cutting in the form of an aperiodic component of the first or second order to perform as the dynamic characteristic of cutting (Eliasberg \& Binder, 1989).

Among the displacements in the system of mechanical processing it is always possible to isolate a group - the one of primary importance in the process of forming the shape of the work-piece. To this group we can attribute the coordinate, which determines the displacement of the tool along the normal path towards the surface of the workpiece at the form-shaping point - the point, which determines the dimensional characteristic of the product in this position.

Tangential displacements, as a rule, do not significantly affect the process of forming nor, as studies have shown, the stability of the cutting process. During the construction of the discrete model it can be the case that the nodal points do not coincide with the form-shaping point. However, it is known that a displacement in the system leads to a change in the thickness and width of shear, which are responsible for the area of shear. Thus, mutual displacements can be accounted for by a change in the area of the shear (Olt et al., 2011).

Using the concept of the delay of the cutting force $P$ with respect to the normal displacement for the description of dynamic properties of the cutting process, the dynamic characteristic of cutting can be written down in the following form:

$$
T_{P} \cdot \dot{P}+P=-k \cdot u_{P}
$$

where $P$ - the dynamic cutting force; $T_{p}$ - the time constant of delay; $k$ - the coefficient of disturbance; $u_{p}-$ a change in the area of shear.

Let us include in our examination two generalized subsystems - that of the workpiece and cutting tool. The subsystem of the work-piece includes the mounting system, adaptation, and the work-piece. The subsystem of the cutting tool includes the mounting system, the system of fastening and the tool. Connection between the subsystems is expressed by the operator of cutting reflecting the interaction of workpiece and cutting tool, whereas the global model of the system is autonomous.

The intuitive-topological analysis of the results of experimental studies enables us to obtain the preliminary simplified model. Taking it into account that a turning system 
is weakly dissipative, and the frequency range of the disturbances is limited, it is possible, within the framework of linearized dynamic models, to accomplish the estimation of the equivalence of the passage from the global system to the model of small dimensionality, which in the present study was carried out by the numerical methods of search employing the procedure of prof. B. L. Veitz (1972). For the analysis of the processes proceeding in the zone of chip formation, it is necessary, within the framework of the proposed research methods, to construct the optimum equivalent model of small dimensionality, which in this study was also carried out by the numerical methods of search.

The need for a passage from the global model of a processing system to more simplified models, which are built according to the hierarchical principle, is accounted for the following basic considerations:

1. With the contemporary facilities of computer technology the structural complexity of the model and the large number of generalized coordinates necessary to achieve the discreteness of the system on the basis of the Finite Element Methods (FEM) is not the principal moment, constituting the need for a passage to simplified models. However, it is also here that we can only use effective computing circuits and algorithms, which limit the accumulation of errors, which can substantially distort the process being investigated.

2. In the limited frequency range the initial global model of a turning system can be infinitely closely represented by the simplified dynamic model with the appropriate identity of amplitude-phase-frequency characteristics (APFC). The above suggestion is based on the idea about the proximity of the frequencies of self-excited oscillations, characteristic of a turning system, to its natural frequencies as a result of the weak dissipativity. If we limit ourselves to the approximate construction of the limits of the region of stability in the space of parameters of the system on the basis of the first method of Lyapunov, then the simplified model, built on the basis of the principle indicated, will prove to be completely correct for finding the solution to the optimization problem by the criterion of maximum productivity of mechanical processing with corresponding limitations. Such natural constraints include durability of the cutting tool, dynamic accuracy and quality coefficients of the surface layer.

In connection with that presented above, the search for the approximating systems was achieved on the basis of the substantiated measure of proximity with the hierarchical complication of the structure of the simplified model. The formation of such structures is based on the balance between deformation and contemporary concepts about the self-excited properties of the system of mechanical processing.

To the matrix of frequency characteristics $W(j \cdot \omega)$ of the refined complex mathematical model of a turning system shown Fig. 1. let us assign a set of values $\{\boldsymbol{W}\}$ for different frequencies $\omega_{i}$ :

$$
W=\left\{W_{1}, W_{2}, \ldots, W_{p}\right\}
$$

where $W_{i}=W\left(j \cdot \omega_{i}\right)-$ the matrix; $i=1,2, \ldots, p ; j=\sqrt{-1}$. 
Set (6) is a sampling from the continuous functional matrix $W\left(j \cdot \omega_{i}\right)$, whereas it is assumed that the sampling is presented without significant losses of information about the behavior of the system. Set (6) was determined experimentally, employing the procedure of that given in the work (Vavilov, 1963).

For the mechanical system in question it is necessary to find the mathematical model, which most adequately reflects the interrelations between the tool and the work-piece in the process of chip formation and is represented by the system of linear differential equations with constant coefficients, which will in a sense be an equivalent general model of a system, but that of lower order (Vavilov, 1963).

The motion of the simplified model of the system of mechanical processing can be represented by the system of differential equations of $m$-th order (whereas $m<n$, where $n$ is the order of the system of equations of the initial model) in the form

$$
\begin{aligned}
& \dot{q}=D \cdot q+G \cdot U \\
& y=C \cdot q
\end{aligned}
$$

where $q-m$ is the measured vector function of the generalized coordinates of the system; $y-k$ is the measured vector function of the outputs of the system; $U-l$ is the measured vector function of disturbances; $D-(m \times m)$ is the matrix of the coefficients of the simplified model; $G-(m \times l)-$ and $C-(k \times m)$ is the assigned matrices.

Applying to the system of equations (7) and (8) the direct one-sided Laplace transform with the zero initial conditions, we will obtain the transmitting matrix of the simplified model in the form

$$
\tilde{W}(p)=C \cdot(E \cdot p-D)^{-1} \cdot B
$$

where $\tilde{W}(p)-(k \times l)$ is the matrix; $E-(m \times m)$ is the unit matrix. Assuming $p=j \cdot \omega_{i}$, we will obtain the matrix of the frequency characteristics of the simplified model above the field of complex numbers, the elements of which are determined by the following dependencies

$$
\widetilde{w}_{u, v}\left(j \cdot \omega_{i}\right)=m_{u, v}\left(\omega_{i}\right) \cdot \exp \left(j \cdot \varphi_{u, v}\right)
$$

$u=1,2, \ldots, k ; v=1,2, \ldots, l$, where $m_{u, v}\left(\omega_{i}\right)=\left|\widetilde{w}_{u, v}\left(j \cdot \omega_{i}\right)\right| ; \varphi_{u, v}\left(\omega_{i}\right)=\operatorname{Arg} \widetilde{w}_{u, v}\left(j \cdot \omega_{i}\right)$.

Matrices $M\left(\omega_{i}\right)$ with the elements $m_{u}, \Phi\left(\omega_{i}\right)$ and $\varphi_{u, v}\left(\omega_{i}\right)$ with the elements of $\varphi_{u, v}\left(\omega_{i}\right)$ are called the matrix of amplitude-frequency and the matrix of phase responses (AFR and APR), respectively.

Determination 1. Two systems are called equivalent in a certain region of frequencies $\Omega$, if the matrices of their frequency characteristics are close for all $\omega \in \Omega$. 
The degree of proximity of the matrices of frequency characteristics is determined by the certificate $\rho(W, \tilde{W})$, which assigns a distance between $w$ and $\tilde{W}$. For the study of complex linear spaces investigated hereby, the following certificates can be accepted:

a) the uniform certificate $(u=1,2, \ldots, k ; v=1,2, \ldots, l)$

$$
\rho_{1}(W, \tilde{W})=\sum_{i=1}^{z} \max \left|\begin{array}{l}
w_{u, v}\left(j \cdot \omega_{i}\right)- \\
\tilde{w}_{u, v}\left(j \cdot \omega_{i}\right)
\end{array}\right|
$$

b) the quadratic certificate

$$
\left.\rho_{2}(W, \tilde{W})=\sum_{i=1}^{z} \sqrt{\Phi_{i} \cdot\left[w_{u, v}\left(j \cdot \omega_{i}\right)-\widetilde{w}_{u, v}\left(j \cdot \omega_{i}\right)\right.}\right]
$$

where $\Phi_{i}=S p\left[w_{u, v}\left(j \cdot \omega_{i}\right)-\widetilde{w}_{u, v}\left(j \cdot \omega_{i}\right)\right]^{T}-$ the track of the matrix $\left[w_{u, v}\left(j \cdot \omega_{i}\right)-\widetilde{w}_{u, v}\left(j \cdot \omega_{i}\right)\right]^{T}$, transposed for $\left[w_{u, v}\left(j \cdot \omega_{i}\right)-\widetilde{w}_{u, v}\left(j \cdot \omega_{i}\right)\right] ; z-$ the number of frequencies entered in the sampling. A scalar function is used as the measure of proximity of the matrices of frequency characteristics

$$
\rho(W, \tilde{W})=\sum_{i=1}^{z} P\left(\omega_{i}\right) \cdot\left[\begin{array}{l}
\operatorname{Sp} G\left(\omega_{i}\right) \cdot G^{T}\left(\omega_{i}\right)+ \\
\operatorname{SpH}\left(\omega_{i}\right) \cdot H^{T}\left(\omega_{i}\right)
\end{array}\right]
$$

where $P\left(\omega_{i}\right)$ - the weighting function; $G\left(\omega_{i}\right), H\left(\omega_{i}\right)$ - $(k \times l)$ - matrix with the elements

$$
\begin{aligned}
& g_{u, v}\left(\omega_{i}\right)=\sigma_{u, v}\left[m_{u, v}\left(\omega_{i}\right)-\tilde{m}_{u, v}\left(\omega_{i}\right)\right] \\
& h_{u, v}\left(\omega_{i}\right)=\chi_{u, v}\left[\varphi_{u, v}\left(\omega_{i}\right)-\varphi_{u, v}\left(\omega_{i}\right)\right]
\end{aligned}
$$

Determination 2. Two systems are called equivalent, if the following condition is satisfied

$$
\rho(W, \tilde{W}) \leq \varepsilon
$$

where $\varepsilon$ - the small predetermined quantity. Fulfillment of conditions (16) ensures the proximity AFR and APR of the generalized and simplified models.

Determination 3. Simplified model with the matrix of coefficients $D^{*}$ of sizes $(m \times l)$ and the corresponding matrix of the frequency characteristics $\tilde{W}^{*}$ is called the optimum model of the $m$ - th order, if the following condition is satisfied 
Madissoo, M.; Maksarov, V.; Arak, M. \& Olt, J.: Dynamic Modeling of Metal Tur...

$$
\rho\left(W, \tilde{W}^{*}\right)=\min \rho(W, \tilde{W})
$$

On the basis of definitions 2 and 3 we assumed that the simplified model of system with the matrix of coefficients $D^{*}$ is the optimum equivalent model of $m$ - the order, if the following condition is satisfied

$$
\rho\left(W, \tilde{W}^{*}\right) \leq \varepsilon .
$$

The analytical solution of the problem of finding the optimum equivalent model in the general case is impossible. Therefore, it is expedient to perform its construction by the numerical methods of search. It is recommended to start the process of search for the optimum equivalent model with the simplest single-mass two-circuit model. If in this case conditions (16), (18) are not satisfied, it is necessary to pass on to models of more complex structure.

Models are complicated due to the inclusion of the new generalized coordinates and inert blocks. The selection of the elastic-dissipative and inert characteristics of the simplified models is accomplished on the basis of the methods of search for extremes in the vector spaces. Employing this procedure for the construction of the equivalent simplified model made it possible to determine not only the elastic and inert parameters of the simplified model, but also to obtain the values of the dissipative parameters for the analysis of the dynamic system.

As an example we examined the workings of turning a rotor shaft BP 3000 with the following dimensions of workpiece - the diameter of $650 \mathrm{~mm}$ and length of $3750 \mathrm{~mm}$ and those of the tools $-H \times B 70 \times 65 \mathrm{~mm}$ on the lathe of model 1M665. The initial model in this case for the subsystems "workpiece" and "tool" had $n_{z g}=319$ units and $n_{u}=420$, respectively. Fig. 2 . presents a standard amplitude-frequency characteristic of subsystems - workpiece (curve - 1) and tool (curve - 2).

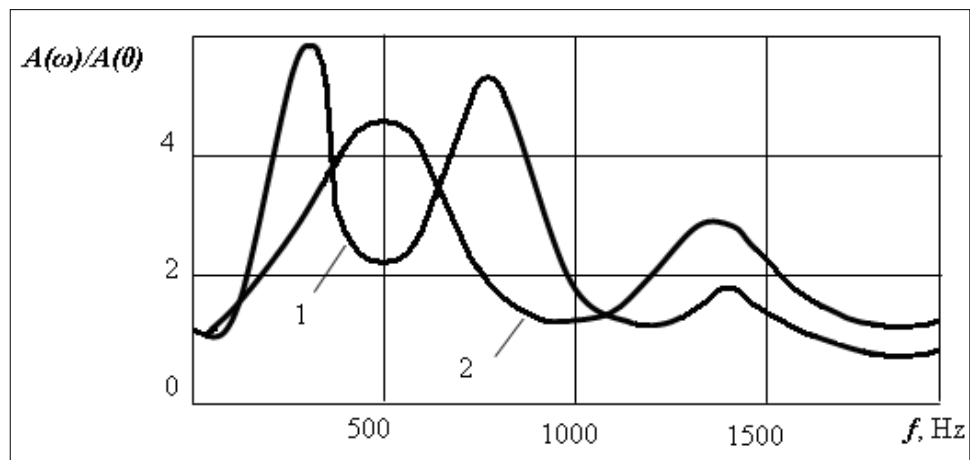

Fig. 2. Amplitude-frequency characteristics of the subsystems of work-piece (curve 1) and tool (curve 2), where A (0) - the static deformation

The simplified equivalent model of work-piece produced had a number of generalized coordinates $q_{z g}=3$ and the model of tool $-q_{u}=2$. Analysis of the dynamic parameters enabled to regard the workpiece as the subsystem of small hardness, and the tool as the subsystem of average hardness (according to the 
classification accepted by Kudinov, V. (1974). The dynamic indices of the technological system in question are located in the limited frequency range of $0 \ldots 1500 \mathrm{~Hz}$. Thus, there is no need to examine higher frequencies, which have an effect on the quality of the workable surface and on the durability of the cutting tool. On the basis of the specific measures of proximity of the generalized and simplified models, and also of evaluation of the amplitude-frequency characteristics of the subsystems of workpiece and tool, for the analysis of the process of chip formation and its influence on the behavior of the system of mechanical processing it is proposed to use a four-contour model of small dimensionality with dissipative characteristics, which account for the structural damping and rheological processes of the global model in question, to which the two subsystems with four generalized coordinates correspond Fig. 3. : 1 - the subsystem of work-piece $B$ with the coordinates $u, w ; 2$ - the subsystem of tool $T$ with the coordinates $x, y$.

Thus, we hereby propose a procedure for the construction of the dynamic model of a turning system as a closed potentially self-excited vibration system allowing for maximum possible completeness of inert and elastic-dissipative properties in the system.

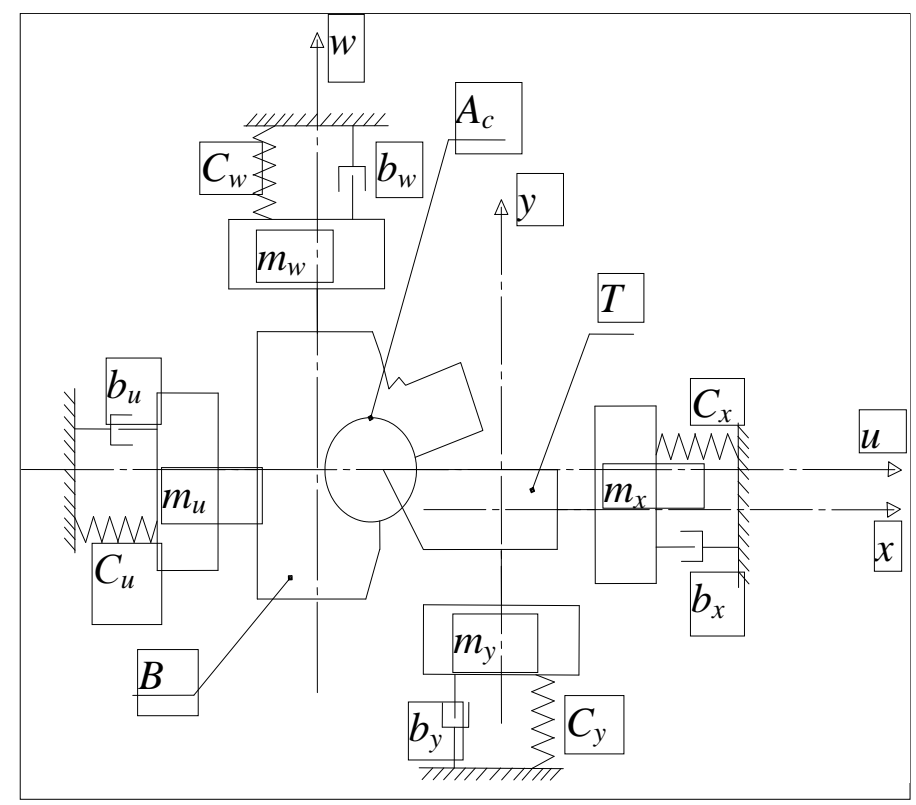

Fig. 3. The dynamic model of the four-contour system (MTFW): B - subsystem "workpiece"; $\mathrm{T}$ - tool; $\mathrm{A}_{\mathrm{c}}$ - the operator of chip formation

\section{Conclusions}

Hereby we propose a procedure for the construction of the dynamic model of a processing system as a closed potentially self-excited vibration system allowing for a maximum possible completeness of inert and elastic-dissipative properties of a turning system. Passage to a simplified dynamic model, equivalent to the relatively spectral characteristics of the initial one (refined - multidimensional), applying the substantiated criterion of proximity in the assigned frequency range enables to construct the limits of the region of stability in the space of processing parameters. 
Madissoo, M.; Maksarov, V.; Arak, M. \& Olt, J.: Dynamic Modeling of Metal Tur...

The future work is to carry out a thorough experimental verification of the here developed model. For this, we have launched a comprehensive series of experimental tests, which results are planned to publish in a future. The ultimate objective is to create a model that fits to describe materials with poor machinability.

\section{References}

Dundur S. T., Das N.S. (2008) Slipline field modeling of orthogonal machining for a worn tool with elastic. Effects and adhesion friction at the contact regions, 1, $2-8$

Eliasberg M. E., Binder M.G. (1989) Increased stability of self-oscillatory system under periodic variation of cutting speed. Mashines and tools, 10, 19-21.

Eliasberg M. E. (1993) Self-oscillations of machine tools. Theory and practice, OKBS, 180 p. (in Russian)

Kovačič I. (1998) The chatter vibrations in metal cutting theoretical approach, Mecahnical Engineering 1, (5), 581 - 593

Kudinov V. A. (1967) Dynamics of machines. Moskow, Mashonostoenije, 359.

Kudinov V. A., Blinov L.I. (1974) Score vibration lathe from the experimental frequency characteristics. Mashines and tools, 6, 6-9 (in Russian)

Li, H.; Zheng, H.; Tang, L. (2010). Theoretical and experimental investigation of the effects of an irregular-pitch cutter on vibration in face-milling. Journal of Mechanical Science and Technology 24 (11) 2169-2174, ISSN: 1738-494X

Murashkin L. S., Murashkin S. L. (1977) Applied nonlinear mechanics tools. Leningrad, Mashinostroenije, 192 (in Russian)

Olt J., Madissoo M., Maksarov, V.V. (2011) Dynamic Stabilization of Technological Systems for Processing Edge Cutting Through the Local Metastability. IEEE International Symposium on Assembly and Manufacturing, $25^{\text {th }}-27^{\text {th }}$ May 2011, Tampere, Finland, 6p. ISBN: 978-1-61284-341-4

Ozel T. (2006) The influence of friction models on finite element simulations of machining. International Journal of Machine Tools and Manufacture, 46, 518530

Panovko J. G. (1960) The internal friction during the vibrations of elastic systems. Moskow, Fizmatgiz. - 193 p. (in Russian).

Podporkin V. G. (1959) Processing of non-rigid parts. Moskow, Mashgish, 208 (in Russian)

Sokolovski A. P. (1958) Vibration at work on metal cutting machines. Study of fluctuations in metal cutting. - Moskow, Mashgish, 3-23 (in Russian).

Stephenson, David A. John S. Agapioa (2006) Metal cutting theory and practice 2nd. ed., by Taylor \& Francis Group, LLC, 825

Vavilov A. A. (1963) Experimental determination of the frequency characteristics of automatic. Energoizdat, 252. (in Russian)

Veitz V. L., Martõnenko A.M. (1972) Self-oscillations in mechanical piecewise linear systems. Nonlinear oscillations and transients in machines. Moskow, Nauka, 283-294 (in Russian) 